\title{
Stent-assisted detachable coil embolization of wide-necked renal artery aneurysms
}

\section{Umberto Marcello Bracale \\ Donatella Narese \\ Ilaria Ficarelli \\ Mario De Laurentis \\ Flavia Spalla \\ Ettore Dinoto \\ Gaetano Vitale \\ Domenico Solari \\ Guido Bajardi \\ Felice Pecoraro}

From the Department of Vascular and Endovascular Surgery (U.M.B. $\triangle$ umbertomarcello.bracale@ unina.it, M.D.L., F.S., G.V.) and Neurosurgery Unit (D.S.), University Federico II of Naples, Naples, Italy; Department of Radiology (D.N.), DIBIMEF and Vascular Surgery Unit (E.D., G.B., F.P.), University of Palermo, Palermo, Italy; Vascular Surgery Unit, (I.F.) AORN Cardarelli, Naples, Italy.

Received 26 November 2015; revision requested 7 January 2016; revision received 7 March 2016; accepted 18 April 2016

Published online 17 November 2016. DOI 10.5152/dir.2016.15551

\begin{abstract}
Renal artery aneurysms (RAAs) are rare with an estimated incidence of $0.1 \%$ in the general population, and they represent approximately $25 \%$ of all visceral aneurysms. The gold standard of treatment is open surgery, but it is associated with a high risk of nephrectomy, mortality, and morbidity. Less invasive endovascular therapies are becoming increasingly common for the treatment of RAAs. Here, we aimed to report three cases of wide-necked complex renal artery aneurysms treated endovascularly using stent-assisted coil embolization with self-expandable stent nitinol Solitaire $A B$ and Concerto Axium coils. In addition, we describe the use of the waffle-cone technique in a case of wide-necked saccular RAA involving the renal artery bifurcation. Technical success was achieved in all three cases with no early or late complications and no recurrences.
\end{abstract}

$\mathrm{R}$ enal artery aneurysms (RAAs) are relatively rare with an estimated incidence of $0.1 \%$ in the general population, rupture incidence of about $30 \%$, and a consequent death rate of $80 \%$ (1). The gold standard of treatment is open surgery, but it is associated with a high risk of nephrectomy (29\%), mortality (1.6\%), and morbidity (12\%). Nowadays, an endovascular approach such as coil embolization or stent-graft with coil embolization is an alternative in the treatment of narrow neck RAAs; however, when facing complex wide-necked aneurysms or complex aneurysm bifurcation, a surgical aneurysmectomy may be required. Recently, stent-assisted coil embolization with preservation of renal blood flow, has become a realistic alternative to surgery in wide-necked, saccular or extraparenchymal aneurysms.

The aim of this study is to report our experience in the treatment of wide-necked complex RAAs through the stent-assisted detachable coil embolization technique in three patients. One case required a stent-assisted coil embolization with waffle-cone technique due to a wide-necked bifurcation RAA.

\section{Technique}

Laboratory investigations including complete blood count, renal and liver function tests, electrocardiography, chest radiography, and ultrasonographic evaluation of the carotid artery, abdominal aorta, and visceral arteries were carried out prior to the procedures. All aneurysms were treated under local anesthesia using a transfemoral approach. After positioning the femoral sheath, an intravenous bolus of $5000 \mathrm{IU}$ of heparin was dispensed. Stent-assisted coil embolization was performed using the Solitaire $A B$ stent nitinol self-expandable electrolytic detachment (Covidien-EV3) and Concerto Axium coils controlled release system (Covidien-EV3). Because of its featured trait of a closed-cell stent with high radial force, Solitaire AB stent does not permit the coils' prolapse and migration, preserving a good blood flow. On the other hand, detachable coils are repositionable, allowing an extremely precise deployment and subsequent embolization of different size aneurysms. After the procedure, the patients were monitored for 48 hours and were discharged with the administration of double antiplatelet therapy, which included acetylsalicylic acid ( $100 \mathrm{mg}$ daily) and clopidogrel (75 mg daily) for six months. After six months, clopidogrel treatment was interrupted and only acetylsalicylic acid (100 mg daily) was maintained.

\section{Case 1}

A 64-year-old man was admitted to the outpatient clinic for evaluation of a left saccular RAA, diagnosed on CT-scan during routine follow-up of left hemicolectomy for colorectal 
cancer. His past medical history was remarkable for hypertension, chronic obstructive pulmonary disease, and hypothyroidism on standard replacement treatment with levothyroxine, along with active smoking. The CT scan showed a saccular aneurysm of $23 \times 25 \mathrm{~mm}$, not involving the branches of the subsequent vascular divisions (Fig. 1a). In the operating room, abdominal aortography was followed by selective left renal arteriography with a $4 \mathrm{~F}$ Berenstein catheter (Boston Scientific). A $45 \mathrm{~cm} 7 \mathrm{~F}$ flexor sheath (Cook Medical) introducer was gently advanced intraluminally into the left renal artery. A microguidewire was then introduced distally to the aneurysm and a $6 \times 30 \mathrm{~mm}$ Solitaire $A B$ stent was advanced. Before deploying the stent, the embolization Rebar 27 reinforced microcatheter (Covidien-EV3) was navigated into the aneurysm and five Concertum Axium detachable coils were released $(16 \mathrm{~mm}$ $\times 20 \mathrm{~cm} ; 14 \mathrm{~mm} \times 30 \mathrm{~cm} ; 12 \mathrm{~mm} \times 30 \mathrm{~cm}$; $10 \mathrm{~mm} \times 30 \mathrm{~cm} ; 10 \mathrm{~mm} \times 30 \mathrm{~cm}$ ) in order to exclude the aneurysm. Once the stent was fully deployed, a completion angiogram was carried out (Fig. 1b), and complete occlusion of the aneurysm with preservation of the renal artery and its branches was established (Fig. 1c). Two days after the procedure the patient was discharged in good clinical condition, without presenting any local or general complication. One week after discharge, the patient's serum creatinine level was unchanged. One-year postembolization, the patient remained asymptomatic with stable renal function (creatinine $0.9 \mathrm{mg} / \mathrm{dL}$ ), and follow-up CT showed no signs of aneurysm recanalization or occlusion of the renal branches either proximally or distally to the aneurysm (Fig. 1d).

\section{Case 2}

A 79-year-old male with hypertension and hepatitis $C$ virus-related liver disease

\section{Main points}

- Treatment of wide-necked renal artery aneurysms remains challenging despite advancement of devices and techniques.

- Stent-assisted detachable coil embolization permits precise and safe exclusion of these complex aneurysms.

Waffle-cone technique, a neurointerventional technique where distal end of a self expandable stent is placed into the base of the aneurysms and coiled through it, is a feasible method to treat bifurcation aneuryms.
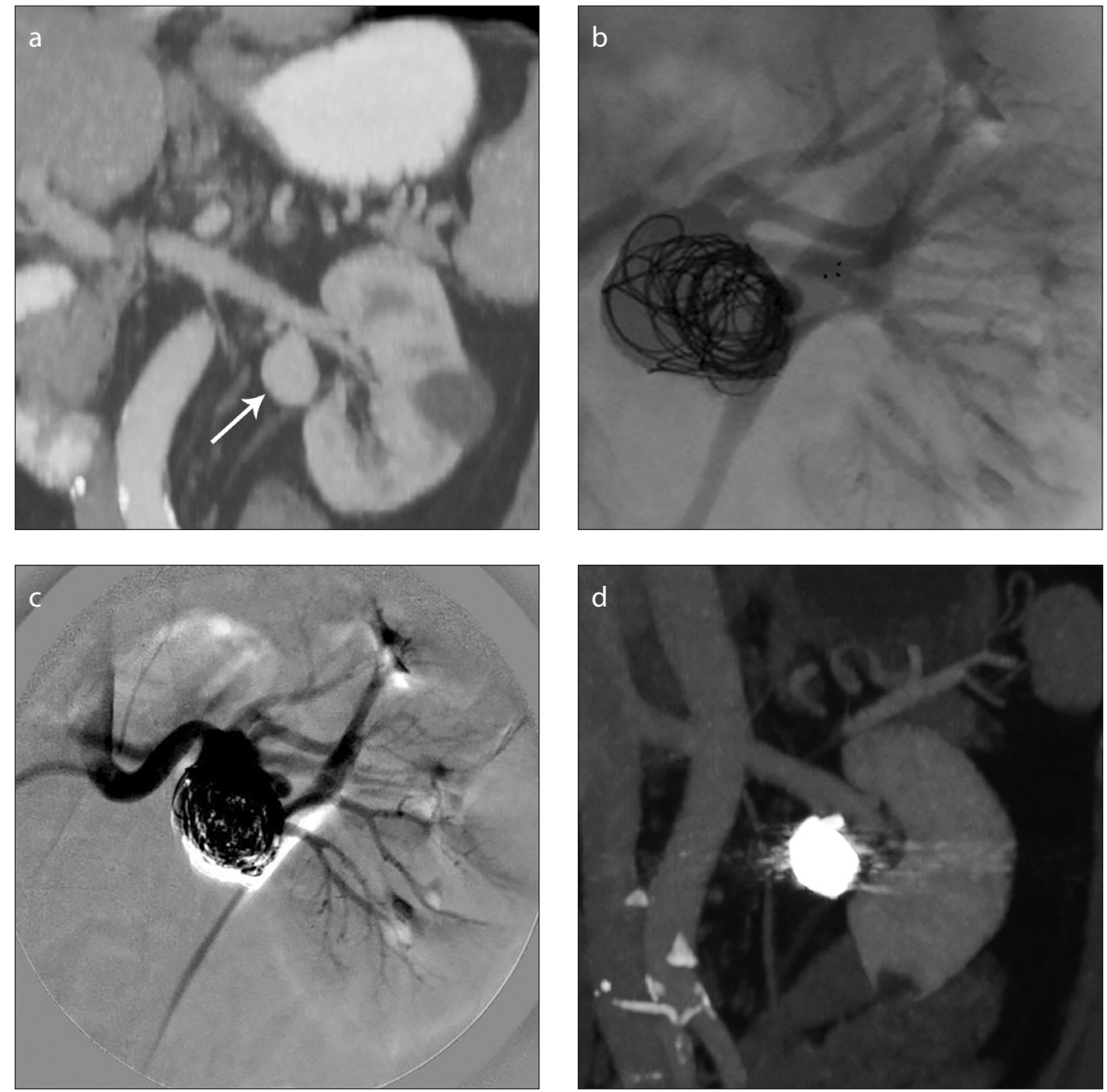

Figure 1. a-d. Preoperative multiplanar reconstruction-computed tomography (MPR-CT) (a) shows a $23 \times 25 \mathrm{~mm}$ renal artery aneurysm (arrow). Intraoperative image (b) demonstrates stent-assisted coil embolization. Completion angiogram (c) shows the aneurysm exclusion and normal kidney perfusion. Twelve-month follow-up MPR-CT scan (d) demonstrates the aneurysm exclusion and valid kidney perfusion.

was admitted for abdominal pain due to gallbladder stones. An abdominal CT-scan revealed a left upper pole renal artery aneurysm of $27 \times 22 \mathrm{~mm}$ (Fig. 2a) with normal perfusion of the renal parenchyma (creatinine $0.84 \mathrm{mg} / \mathrm{dL}$ ). After selective left renal artery angiography, we proceeded to guide catheterization with 0.014-inch Pilot (Abbott) first of the renal artery branch and then of the aneurysm itself. Next we advanced a Solitaire $A B$ stent $6 \times 30 \mathrm{~mm}$. Before deploying the stent, the embolization Rebar 27 reinforced microcatheter was navigated into the aneurysm and four Concerto Axium coils (18 $\mathrm{mm} \times 40 \mathrm{~cm} ; 12 \mathrm{~mm} \times 30 \mathrm{~cm} ; 20 \mathrm{~mm} \times 50$ $\mathrm{cm} ; 6 \mathrm{~mm} \times 20 \mathrm{~cm}$ ) were released in order to exclude the aneurysm. At last, the stent was fully deployed at the plan of the arterial segment over the aneurysm neck and a completion angiogram was performed, which showed the complete exclusion of the aneurysm. The patient did not have any symptoms or clinical complications. One year after the endovascular treatment, follow-up CT showed complete thrombosis of the aneurysm with preservation of renal blood flow. Blood pressure and renal function remained stable (creatinine $0.89 \mathrm{mg} /$ $\mathrm{dL}$ ), and the patient remained asymptomatic throughout the follow-up (Fig. 2b).

\section{Case 3}

A 40-year-old male presented with right RAA, discovered accidentally during abdomen imaging. The patient's history was negative for common cardiovascular risk factors; laboratory findings showed normal renal function with no indications for hematuria or proteinuria. CT angiography confirmed presence of a wide-necked right renal artery bifurcation aneurysm with a diameter of $28 \times 24 \mathrm{~mm}$ (Fig. 3a).

Under local anesthesia, a $55 \mathrm{~cm} 5 \mathrm{~F}$ flexor sheath was placed in the right common femoral artery. The right renal artery was then catheterized using a $5 \mathrm{~F}$ Cobra catheter and 

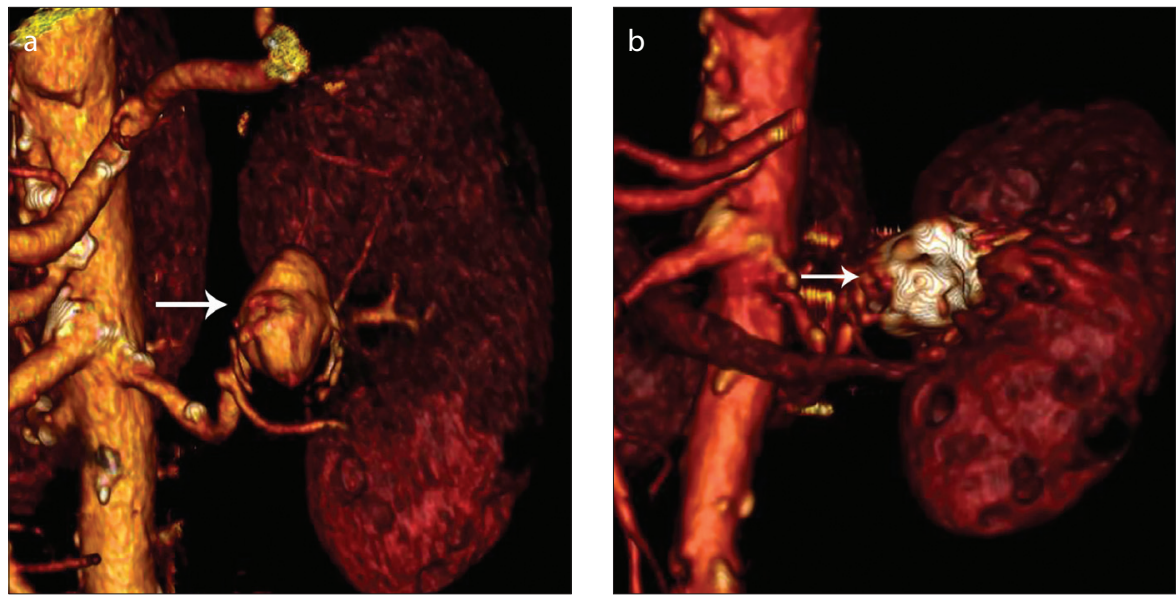

Figure 2. a, b. Preoperative volume rendering (VR) CT scan (a) shows a $27 \times 22 \mathrm{~mm}$ renal artery aneurysm (arrow). Twelve-month follow-up VR-CT scan (b) shows the aneurysm exclusion and valid kidney perfusion (arrow).
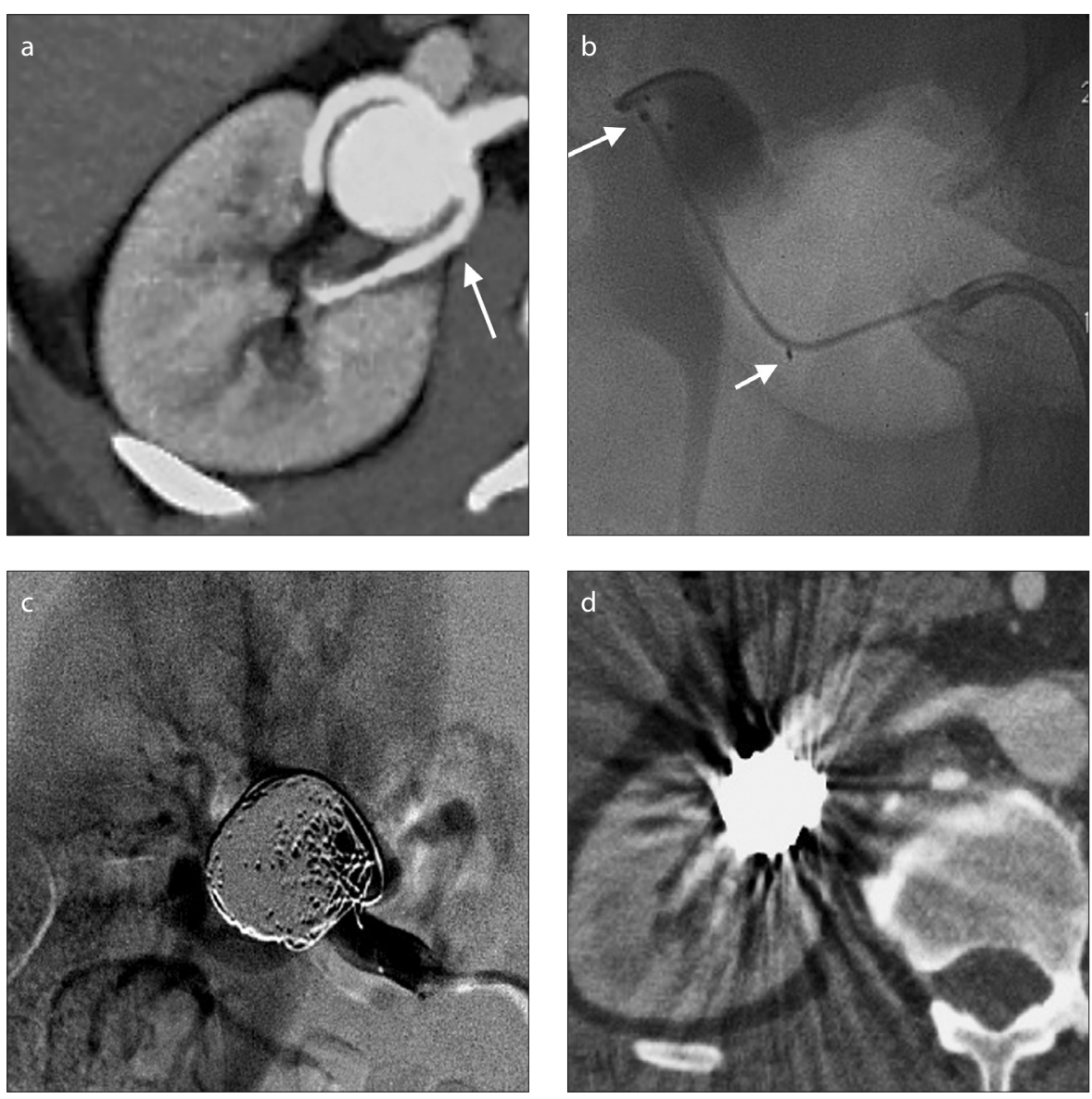

Figure 3. a-d. Preoperative MPR-CT scan (a) shows a $28 \times 24 \mathrm{~mm}$ renal artery aneurysm (arrow). Intraoperative fluoroscopy (b) shows stent deployment (arrow). Completion angiogram after aneurysm coil packaging (c) shows aneurysm occlusion and valid kidney perfusion. Twelve-month follow-up axial CT scan (d) demonstrates the aneurysm occlusion and normal kidney perfusion.

the sheath gently advanced over an Amplatz guidewire. Afterwards, a $6 \times 30 \mathrm{~mm}$ Solitaire $A B$ stent was implanted into the aneurysm intentionally covering the bifurcation. The stent diameter was determined based on the assessment of the size of the aneu- rysm neck and the diameter of the proximal parent artery. In this way, a waffle-cone was created by deploying the stent's proximal edge in the renal artery and the distal one at the proximal extreme of the aneurysm. Such disposition avoids the migration of the coils allowing good flow in the division branches (Fig. 3b). After successful stenting, a rebar 27 microcatheter with coaxial technique was advanced into the aneurysm through the stent's lumen for coiling. Controlled release of three Concerto Axium coils $(20 \mathrm{~mm}$ $\times 50 \mathrm{~cm} ; 18 \mathrm{~mm} \times 40 \mathrm{~cm} ; 18 \mathrm{~mm} \times 50 \mathrm{~cm}$ ) was then performed (Fig. $3 \mathrm{c}$ ). The intra- and postoperative periods were uneventful. Imaging and laboratory examinations showed preservation of renal function (creatinine 1 $\mathrm{mg} / \mathrm{dL}$ ), and patient was symptom-free at six- and twelve-month follow-ups. Angiographic control at six months and CT exam at one year showed good results over the long-term (Fig. 3d).

\section{Discussion}

RAAs are rare, predominantly asymptomatic and usually detected during the workup for hypertension or, incidentally, during abdomen imaging. Multiple etiologies of RAAs are known; most of the cases are due to fibrodysplasia, atherosclerosis, post-traumatic or mycotic origin, Ehlers-Danlos or Marfan syndrome, Takayasu or Behcet disease, and Rechlinghausen neurofibromatosis $(2,3)$. The natural history of RAAs is still unknown, their relationship with hypertension is controversial, and the risk of complications, particularly rapture, has led to adopting the surgical excision as routine treatment. There are still great limits in the comprehension of the true risk of rupture, but a number of different causes have been identified as possible risk factors for such complication, including morphology and histologic characteristics of the aneurysm, gender, menopausal status, and pregnancy $(3,4)$. Currently, the indications for endovascular arterial reconstruction include RAA size greater than $2 \mathrm{~cm}$, renovascular hypertension, embolization, local pain, hematuria, and high-risk patients (women at childbearing age or patients with a single kidney). Most recent data suggest that rupture can occur in aneurysms of less than 1.5 $\mathrm{cm}$ in size, which brings up the question of whether smaller lesions should be treated as well $(3,5,6)$. Overall, aneurysms involving one or more branches have indication for open surgery. Usually open repair requires the aneurysm excision with or without patch angioplasty; however, bypass or interposition graft can be considered as valid alternatives. Distal clamping is not a feasible option when the RAAs grow near the hilus and involve more than one arterial branch to the renal parenchyma. In that 


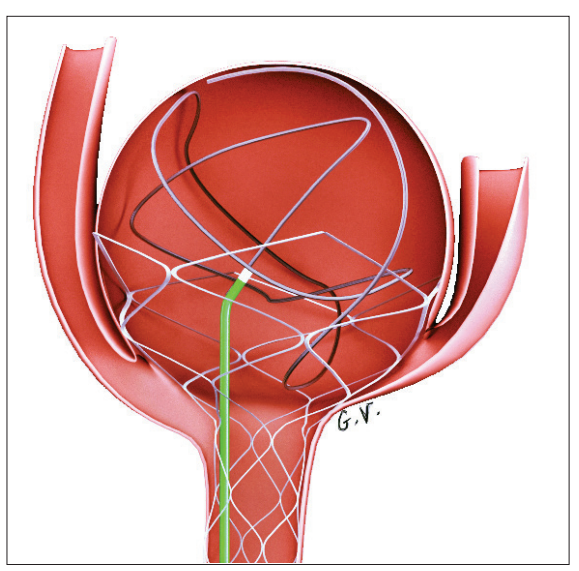

Figure 4. Solitaire AB (Covidien-EV3) device. The device must be advanced through a 0.021-inch microcatheter. Embolic coils are placed through the device's interstices using a microcatheter with a $<2.5 \mathrm{~F}$ tip using accepted coiling practices, taking care of having previously selected a precise framing coil. At the end of the procedure framing coil and stent can be extracted.

case, ex-situ repair becomes a possible approach; this sort of surgery requires either a retroperitoneoscopic approach or a flank incision.

Endovascular repair should be considered if the patient's anatomy is favorable. So far, there are two general endovascular approaches: transcatheter embolization for saccular aneurysms and endovascular stent-grafting for aneurysms that present a sufficient distal and proximal landing zone. Recently, the emergence of new materials and the refinement of endovascular techniques has allowed the introduction of stent-assisted coil embolization or the use of multilayer flow modulator stent, extending the endovascular treatment to multiple types of complex aneurysms $(7,8)$. The most severe complication is the occlusion of the main renal artery due to coil migration. One of our patients presented a complex wide-necked right renal artery bifurcation aneurysm and was therefore treated using the endovascular transcatheter embolization waffle-cone technique to avoid migration. This method represents an evolution of stent-assisted coil embolization and a vanguard approach for wide-necked bifurcation RAAs. In all our cases, we chose the Solitaire AB stent, because it has a closedcell system, prevents protrusion of the coils, adapts better to the anatomy of the vessels due to its flexibility, and presents a low percentage of restenosis. Moreover, it can be completely retrieved even when fully deployed for procedural control, and it has a good radial force and kink resistance, designed for optimal vessel conformability, with enough resistance to coil protrusion (Fig. 4).

The available coil types are either pushable or detachable. Pushable coils are deployed by driving them with a wire or a specific wire-pusher; detachable coils function by controlled-deployment that permits a reposition before the coil's release. Main advantage of detachable coils is the chance of retrieval in case of misplacement, allowing more precise deployment and embolization for various sizes of arteries. Therefore, they are mostly indicated in more complicated cases. On the other hand, they are more expensive and present longer set-up times. Pushable coils are often used in narrow-necked RAAs, while in wide-necked RAAs, given the unfavorable anatomy, the use of detachable coils is safer: this is the reason why we prefer the latter.

Major limitation of our study is the small number of cases $(n=3)$ described here. Although our aim was strictly to observe the short and mid-term results when evaluating RAAs' exclusion by coil embolization, no comparison was done with groups treated either with different types of endovascular techniques or open surgery treatment. Whether RAAs should be treated by open surgery or endovascular repair is still unclear. Devices and interventional techniques that are now applied to the treatment of RAA were first studied and applied to the cure of intracranial wide-necked aneurysms. With such background consideration, neurointerventional procedures and techniques can significantly contribute to the overall technical innovation of endovascular surgery. Despite the promising prelim- inary results of endovascular treatment using stent-assisted coil embolization, and in one case the waffle-cone technique, further studies are needed to confirm its efficacy and demonstrate good long-term outcome.

In conclusion, stent-assisted coil embolization of wide-necked renal artery aneurysms is an evolutionary step in the treatment of RAAs. Our experience indicates that stent-assisted coil embolization, using detachable coils and self-expandable stent nitinol, is technically feasible and effective for the exclusion of challenging widenecked and bifurcation complex RAAs without sacrificing any branch arteries. In addition, our experience showed the waffle-cone technique is a safe, valid alternative tool for stent-assisted coiling of complex wide-necked bifurcation aneurysms that have unfavorable anatomic features for conventional stent assisted coiling.

Conflict of interest disclosure

The authors declared no conflicts of interest.

\section{References}

1 Pfeiffer T, Reiher L, Grabitz K, et al. Reconstruction for renal artery aneurysm: operative techniques and long-term results. J Vasc Surg 2003; 37:293-300. [CrossRef]

2 Eskandari MK, Resnick SA. Aneurysms of the renal artery. Semin Vasc Surg 2005; 18:202-208. [CrossRef]

3 Sédat J, Chau Y, Baque J. Endovascular treatment of renal aneurysms: a series of 18 cases. Eur J Radiol 2012; 81:3973-3978. [CrossRef]

4 Tham G, Ekelund L, Herrlin K, et al. Renal artery aneurysms. Natural history and prognosis. Ann Surg 1983; 197:348-352. [CrossRef]

5 Hupp T, Allenberg JR, Post K, et al. Renal artery aneurysm: Surgical indications and results. Eur J Vasc Surg 1992; 6:477-486. [CrossRef]

6 Reiher L, Brabitz K, Sandmann W. Reconstruction for renal artery aneurysm and its effect on hypertension. Eur J Vasc Endovasc Surg 2000; 20:454-456. [CrossRef]

7 Tsilimparis N, Reeves JG, Dayama A, et al. Endovascular vs. open repair of renal artery aneurysm: outcomes of repair and long-term renal function. J Am Coll Surg 2013; 217:263-269. [CrossRef]

8 Canyiğit $M$, Çam $A$, Çetin $H$, Altunoğlu $A$, Hıdıroğlu M. The use of the Cardiatis multilayer flow modulator stent to treat sequential saccular aneurysms of the renal artery in a solitary kidney. Türk Göğüs Kalp Damar Cerrahisi Dergisi 2015; 23:354-358. [CrossRef] 